\title{
Resource Scheduling Based on Unequal Clustering in Internet of Things
}

\author{
Hongying Bai $\mathbb{D}^{1,2}$ Xiaotong Zhang ${ }^{1},{ }^{1}$ Yingdong Xie, ${ }^{2}$ Haiyan Gong, ${ }^{1}$ Zhuang Li, ${ }^{1}$ \\ and Shilong Liu ${ }^{1}$ \\ ${ }^{1}$ University of Science and Technology Beijing, Beijing 100083, China \\ ${ }^{2}$ Ordos Institute of Technology, Ordos 017010, China \\ Correspondence should be addressed to Xiaotong Zhang; zxt@ies.ustb.edu.cn
}

Received 18 September 2021; Accepted 31 December 2021; Published 25 January 2022

Academic Editor: Nicola Bicocchi

Copyright (C) 2022 Hongying Bai et al. This is an open access article distributed under the Creative Commons Attribution License, which permits unrestricted use, distribution, and reproduction in any medium, provided the original work is properly cited.

\begin{abstract}
Resource scheduling in a fair and efficient manner is a significant challenge in the Internet of Things. Although unequal clustering is an effective technique for alleviating the "energy holes" problem in multihop communication, resource scheduling based on unequal clustering is scarcely conducted. In the present study, a new resource scheduling based on unequal clustering in the Internet of Things (RSUC) is proposed. In RSUC, unequal clustering and multihop routing are considered, and the "energy holes" problem is alleviated effectively. RSUC includes resource scheduling of intracluster communication and intercluster communication. In resource scheduling of intracluster communication, according to the threshold of the number of cluster members, the cluster heads (CHs) recycle resources of failed nodes. In resource scheduling of intercluster communication, $\mathrm{CHs}$ in the different layers based on transmission chain obtain different sending and receiving time slots. In RSUC, CHs that are near the base station (BS) have fewer intracluster communication time slots and more intercluster communication time slots. Clusters that are further away from the BS end intercluster communication earlier and enter into intracluster communication instead of waiting for all $\mathrm{CHs}$ to complete intercluster communication. The simulation results reveal that RSUC significantly increases the network throughput and reduces the energy consumption of the Internet of Things.
\end{abstract}

\section{Introduction}

The Internet of Things (IoT) is commonly regarded as the third wave of the information industry after computers and the Internet. The development trend of the IoT will guide the development direction of information technology. IoT has been applied in various fields such as smart homes, smart cities, industrial automation, intelligent transportation, and healthcare systems [1-3]. For example, the Internet of Things can be applied in smart agriculture [4]. In agricultural monitoring systems completed in reference, the massive time series data are stored by using the time series database InfluxDB, which can monitor the orchard environment and flood conditions [5]. Despite such features, several challenging research areas remain, such as the interoperability of systems, resource scheduling, and energy management.
A significant challenge in data collection of the IoT is resource scheduling of a large number of IoT nodes [6]. Inspired by time slot channel frequency hopping, Yang et al. proposed a time and power resources allocation in the industrial Internet of Things to maximize the energy efficiency of the system and ensure the stability of communication between IoT nodes [7]. Liu et al. investigated a time scheduling scheme for wireless powered ambient backscatter communications in the Internet of Things [8].

Clustering provides effective solutions for reducing energy consumption and extending the lifetime of the IoT. Numerous clustering protocols have been proposed by researchers in previous studies [9]. Aher et al. proposed smart agriculture using clustering and IoT to judge the parameters and present a graphical reading for the users to understand the requirement of the parameters [10]. For multihop communication of large-scale energy-limited wireless 
networks, the nodes closer to the base station (BS) will undertake heavy forwarding work, which will result in "energy holes" [11]. Unequal clustering is an effective technique for alleviating the "energy holes" problem. In unequal clustering, the network is divided into clusters of different sizes, and the size of the clusters far from the BS is larger than that of the clusters close to the BS.

Recently, numerous studies on resource scheduling for the Internet of Things have achieved notable results $[12,13]$. Several researchers focused on resource scheduling based on tree topology. Lee et al. proposed the Tree TDMA MAC algorithm using the allocation of time slots and frequencies [14]. Osamy et al. proposed TDMA scheduling for treebased data collection using a genetic algorithm [15]. Due to the different network characteristics and application environment of various Internet of Things, resource scheduling usually needs to compromise and optimize a variety of design schemes.

In the present study, resource scheduling based on unequal clustering in the Internet of Things (RSUC) is proposed. The main contributions of this paper include the following: (1) Unequal clustering is used to alleviate the "energy holes" problem, and in resource scheduling of intracluster communication, the $\mathrm{CH}$ s dynamically recycle resources of failed nodes. (2) A routing strategy based on unequal clustering and cluster head layering mechanism is proposed. (3) Resource scheduling based on unequal clustering (RSUC) is proposed in this paper. In RSUC, the CHs near the base station have fewer intracluster communication time slots and more intercluster communication time slots, thereby improving network throughput.

The remainder of the present paper is organized as follows. Related work is discussed in Section 2. The network model is given in Section 3. The resource scheduling based on unequal clustering in the Internet of Things is presented in Section 4. The theoretical analysis is given in Section 5. Experiments are discussed in Section 6, and the conclusion is provided in Section 7.

\section{Related Work}

Clustering provides effective solutions to reduce energy consumption in the large-scale IoT. For efficient connectivity and communications in the IoT, clustering of the devices was proposed in previous studies [16, 17]. The strategy of unequal clustering can alleviate the "energy holes" problem in multihop communication. Mohamed et al. proposed the dynamic load-balancing cluster-based protocol (DLCP) [18]. DLCP divides the nodes into unequal size clusters. Compared with clusters farther away from the $\mathrm{BS}$, the closest clusters to the BS have smaller sizes. In the $\mathrm{CH}$ rotation phase, the node with the highest residual energy is selected as the $\mathrm{CH}$ in the next round. In previous research of the present authors, unequal clustering and a routing algorithm based on dynamic topology (DUCR) [19] were proposed, which could balance the network load and prolong the network lifetime. In DUCR, reclustering is performed only when the number of cluster members is beyond the threshold. Clustering is also beneficial for establishing the topology, which could be used to optimize the parameters of quality of service (QoS) and facilitate the management of resources of the heterogeneous Internet of Things [20]. If the nodes are densely distributed in the Internet of Things, the data collected by adjacent nodes will be the same or similar. For clustered networks, the cluster head can fuse data of cluster member. Alam et al. reviewed the data fusion of IoT focusing on mathematical methods and specific environment [21].

Routing protocols generally traverse all neighbor nodes to find a relay node. Hierarchical cluster-based routing approaches are efficient solutions that can prolong network life and reduce network energy consumption. Huynh et al. proposed a routing algorithm based on unequal clustering [22]. Once the $\mathrm{CH}$ s are selected and clusters are formed, $\mathrm{CH}$ with high residual energy and close to BS are selected as parent $\mathrm{CH}$ for all other remaining CHs. In one study [23], a multibranch tree-based clustering and routing approach was proposed, in which each level is designated an independent node set which leads to the formation of the backbone of the tree. Energy conservation is a significant challenge in the Internet of Things. A cluster tree-based routing protocol (CT-RPL) is proposed [24], in which $\mathrm{CH}$ selection is accomplished using a game theoretic approach. Senkar et al. proposed an energy-saving clustering and routing protocol for the Internet of Things based on swarm intelligence [25], which uses a swarm algorithm to select cluster heads and reduce the delay of data to base stations. $\mathrm{Xu}$ et al. proposed an improved LEACH clustering routing algorithm in the perception layer of the Internet of Things [26], which optimized the cluster head selection strategy and the routing between the cluster head and the sink node. Chithaluru et al. proposed energy-saving routing scheduling of the Internet of Things based on fuzzy sorting [27]. The cluster head acts as a relay node to transmit data to the BS.

Resource scheduling can improve the utilization of limited resources and network throughput. S Abdullah et al. proposed double deep Q-learning-based scheduling in the IoT (DDQL) [28], which could achieve an efficient policy in terms of time and cost-saving under the resource and deadline constraints using the deep reinforcement learning approach. Renato et al. proposed a link scheduling algorithm [29], which minimizes the number of time slots required by the link so that nodes can communicate without interference under the SINR model. Gabale et al. proposed the PIP (Packets in Pipe) algorithm [30], in which the allocation of channels is assigned based on the level of nodes. The main idea of PIP allocation is that the nodes in odd levels use odd time slots and the nodes in even levels use even time slots. However, state transitions of sending and receiving in the PIP algorithm are too frequent.

For clustered networks, two-stage scheduling, which is the resource allocation of intracluster and intercluster communication, was proposed for the hierarchical structure of WSN [31]. A graph coloring method is used to allocate channels, and TDMA is used for time slot allocation in intracluster communication. For intercluster communication, according to the network topology information, dynamic programming is adopted to find the maximum set of 
nodes that can be transmitted in parallel. Seedha Devi et al. proposed a data collection scheme based on clustering [32] to reduce delay and packet loss. In the algorithm, the aggregation tree is constructed by the sink node using the minimum spanning tree (MST). Abdullah et al. proposed shortest processing time scheduling (SPT) [33], in which sensors are connected to make IoT groups, and each group has a designated broker. The brokers choose a scheduling strategy to transmit messages to the sink node.

The various aforementioned methods of resource scheduling are not based on the unequal clustering for alleviating the "energy holes" problem, and the problems of reusing and recycling resources of failed nodes are ignored. Several methods of resource scheduling are quite complex, which creates a significant challenge in terms of computational efficiency. In the present study, in considering the requirements of dynamic topology and multihop routing, efficient resource scheduling based on unequal clustering is proposed. In intracluster communication, the cluster heads recycle resources of failed nodes according to the threshold of the number of cluster members. In intercluster communication, $\mathrm{CHs}$ in different layers based on transmission chain obtain different sending and receiving time slots. Clusters that are further away from the BS end intercluster communication earlier and enter into intracluster communication instead of waiting for all $\mathrm{CHs}$ to complete intercluster communication, which improves time slot utilization and network throughput.

\section{Network Model}

In this section, the network model and transmission interference model of RSUC are introduced.

3.1. Network Model. Resource scheduling of a large number of IoT nodes is a significant challenge in data transmission. Dynamic changes of unequal clustering and multihop routing are comprehensively considered in the present resource scheduling. Each cluster has a cluster head $(\mathrm{CH})$ that collects the data from cluster members (CM) and forwards data to relay $\mathrm{CH}$ in a hop-by-hop manner, which continues until the data arrives at the base station (BS). Figure 1 shows the network topology after unequal clustering and multihop routing.

The following constraints are met in RSUC:

(1) The nodes can calculate the distance based on the received signal strength indication (RSSI)

(2) The transmission power of nodes is adjustable

(3) The data of CM are generated periodically

(4) The CHs fuse the data received from CM in intracluster communication, but data of the $\mathrm{CH}$ is not fused during intercluster communication

(5) The BS supports multi-interface and multichannel communication

Table 1 lists the symbol representation used in RSUC.

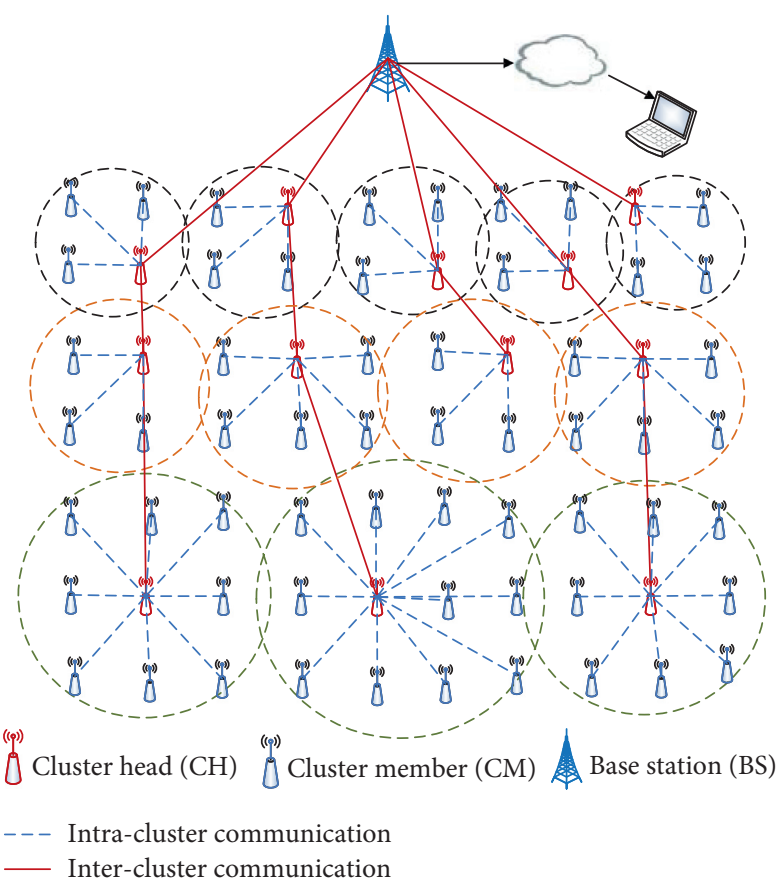

Figure 1: Network topology based on unequal clustering.

3.2. Transmission Interference Model. The premise of resource scheduling is to avoid conflicts while transmitting data. There are two typical transmission interference models, namely, the graph-based protocol model and the SINRbased physical model. RSUC is based on the graph-based protocol model, which includes primary conflict and secondary conflict [34]. The primary conflict of the graph-based protocol model affects the range between one-hop neighbors. For example, as shown in Figure 2(a), A received data of both $B_{1}$ and $B_{2}$. In Figure 2(b), $A$ sent data to $B_{2}$ while receiving data of $B_{1}$. A primary conflict occurs when one node attempts multiple operations at the same time. The secondary conflict of the graph-based protocol model affects the range among the two-hop neighbors, which is usually considered to be a hidden terminal problem. Such a problem can be observed in Figure 2(c), in which the communication between $B_{2}$ and $C$ affected the communication between $B_{1}$ and $\mathrm{A}$ when they used the same channel to transmit. Transmission interference can be avoided by effective resource scheduling with channel and time slot allocation.

\section{Proposed Resource Scheduling Based on Unequal Clustering}

In this section, the resource scheduling based on unequal clustering in IoT (RSUC) is presented. The structure of RSUC is shown in Figure 3.

4.1. Unequal Clustering and Multihop Routing. The research of dynamic unequal clustering and routing was completed in a preliminary study by the present authors [19]. The competition radius of the node in unequal clustering is defined as denoted in 
TABLE 1: Symbol representations of RSUC.

\begin{tabular}{|c|c|}
\hline Symbol & Description \\
\hline $\mathrm{CH}_{\mathrm{i}}$ & Cluster head $i$ \\
\hline $\mathrm{CM}$ & Cluster member \\
\hline FL_CH & $\mathrm{CHs}$ of the first level \\
\hline $\mathrm{E}_{\mathrm{CH} i}^{-}$ & Residual energy of $\mathrm{CH}_{i}$ \\
\hline $\mathrm{D}\left(\mathrm{CH}_{i}, \mathrm{BS}\right)$ & Distance from $\mathrm{CH}_{i}$ to $\mathrm{BS}$ \\
\hline $\mathrm{NBr}_{n i}$ & Neighbors of $n_{i}$ \\
\hline $\mathrm{N}_{-} \mathrm{NBr}_{\mathrm{CH} i}$ & Number of neighbor nodes of $\mathrm{CH}_{i}$ \\
\hline $\operatorname{Cost}\left(\mathrm{CH}_{\mathrm{i}}, \mathrm{CH}_{\mathrm{j}}\right)$ & Cost function of the $\mathrm{CH}_{i}$ to $\mathrm{CH}_{j}$ \\
\hline $\mathrm{RN} \_\mathrm{CH}_{i}$ & Relay node of $\mathrm{CH}_{i}$ \\
\hline CRN_CH & Candidate relay node of $\mathrm{CH}_{i}$ \\
\hline $\mathrm{CH}_{\mathrm{i}}{ }_{\mathrm{h}} \mathrm{pp}$ & Number of hops from $\mathrm{CH}_{i}$ to BS \\
\hline$L_{i}$ & Level $i, L_{\max }$ represents the highest level in the IoT \\
\hline $\mathrm{CH}_{\mathrm{Li}}$ & The set of $\mathrm{CH}$ in $L_{i}$ \\
\hline $\mathrm{LCH}_{i}$ & Level of $\mathrm{CH}_{i}$ \\
\hline $\mathrm{N}_{-} \mathrm{CH}_{\mathrm{Li}}$ & Number of $\mathrm{CHs}$ in set $\mathrm{CH}_{\mathrm{Li}}$ \\
\hline N_CM & Number of cluster members \\
\hline$T_{\mathrm{N} \_\mathrm{CM}}$ & Threshold of number of cluster members \\
\hline TD_CM & Amount of data in cluster member \\
\hline$S$ & Sending state \\
\hline$R$ & Receiving state \\
\hline$C_{i}$ & Channel $i$ \\
\hline TS_CH $\mathrm{CH}_{i}$ & Time slot of $\mathrm{CH}_{i}$ \\
\hline TTS & Total time slot of intercluster communication \\
\hline EOD_CH ${ }_{i}$ & End of data in $\mathrm{CH}_{i}$ \\
\hline
\end{tabular}

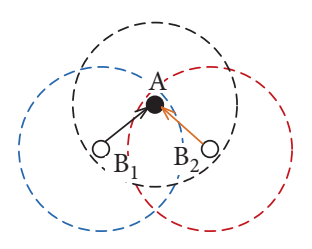

(a)

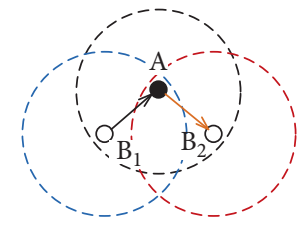

(b)

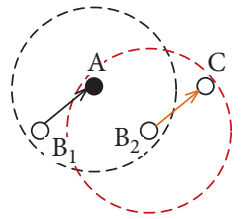

(c)

FIgURE 2: Transmission interference model.

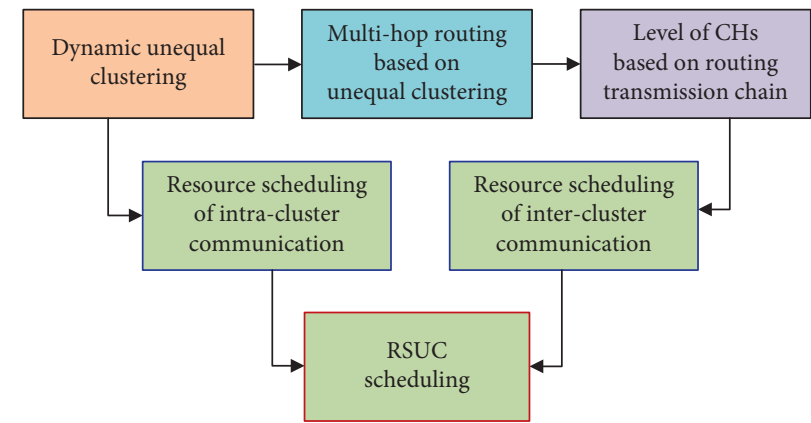

Figure 3: Structure of RSUC.

$$
\begin{aligned}
n_{i} \cdot R_{c}= & \left(1-\mu 1 \frac{D_{\max }-D\left(n_{i}, B S\right)}{D_{\max }-D_{\min }}\right)\left(1-\mu 2 \frac{1}{\left|N B r_{n i}\right|}\right) \\
& \left(1-\mu 3 \frac{E_{n i_{\text {_init }}}-E_{n i}}{E_{\text {ni_init }}}\right) R_{c}^{0},
\end{aligned}
$$

where $D_{\max }$ represents the maximum distance from the node to the BS, $D_{\min }$ represents the minimum distance from the node to the $\mathrm{BS}$, and $F_{c}^{0}$ represents the initial maximum competition radius.

After unequal clustering, the cluster size is larger where the distance from $\mathrm{CHs}$ to the $\mathrm{BS}$ is longer, the number of 
neighbors of $\mathrm{CH}$ is smaller, and there is more remaining energy. In RSUC, the $\mathrm{CH}$ of the first level (FL_CH) directly communicates with the BS. The $\mathrm{CH}$ s in $\mathrm{FL}_{-} \mathrm{CH}$ are removed from the FL_CH when the current energy of $\mathrm{CH}$ reduces to the threshold of energy of the first level. If necessary, the supplement of FL_CH is required.

Aside from the $\mathrm{CH}$ s in set $\mathrm{FL} \_\mathrm{CH}$, all other $\mathrm{CH}$ s need to find relay node in multihop routing. The cost function of the relay node of $\mathrm{CH}_{\mathrm{i}}$ is defined as denoted in

$$
\begin{aligned}
\operatorname{Cost}\left(\mathrm{CH}_{i}, \mathrm{CH}_{j}\right)= & \omega_{1} \times I s R_{\mathrm{CH} j}+\omega_{2} \frac{E_{\mathrm{CH}_{i}}}{E_{\mathrm{CH}_{j}}} \\
& +\omega_{3} \frac{C M_{-} \mathrm{Num}_{\mathrm{CH} j}}{\mathrm{CM} \mathrm{Num}_{\mathrm{CH} i}+1} \\
& +\omega_{4} \frac{D\left(\mathrm{CH}_{i}, \mathrm{CH}_{j}\right)+D\left(\mathrm{CH}_{j}, \mathrm{BS}\right)}{D\left(\mathrm{CH}_{i}, \mathrm{BS}\right)} .
\end{aligned}
$$

(1) If $\mathrm{CH}_{\mathrm{i}} \notin \mathrm{FL} \_\mathrm{CH}$ and $\mathrm{CH}_{\mathrm{j}} \in \mathrm{NBr}_{\mathrm{Chi}}$ and $\mathrm{CH}_{i} \notin \mathrm{FL}_{-} \mathrm{CH}$ an $\mathrm{d} \mathrm{NBr}_{\mathrm{CH} i} \cap \mathrm{FL} L_{-} \mathrm{CH}=\varnothing$ and
$\mathrm{CH}_{j} \in\left(\mathrm{NBr}_{\mathrm{CH}_{i}} \cup \mathrm{FL} \mathrm{C}_{-} \mathrm{CH}\right)$, then the value of $\operatorname{Cost}\left(\mathrm{CH}_{\mathrm{i}}, \mathrm{CH}_{\mathrm{j}}\right)$ can be calculated.

(2) If $\mathrm{CH}_{\mathrm{i}} \in \mathrm{FL} \_\mathrm{CH}$, then $\operatorname{Cost}\left(\mathrm{CH}_{\mathrm{i}}, \mathrm{CH}_{\mathrm{j}}\right)=-1$, and the relay node of $\mathrm{CH}_{i}$ is the $\mathrm{BS}$.

(3) The parameters meet the conditions that $\omega_{1}+\omega_{2}+\omega_{3}+\omega_{4}=1$.

IsR $_{\mathrm{CHj}} \in\{0,1\}, 1$ means that $\mathrm{CH}_{\mathrm{j}}$ is a relay node of $\mathrm{CH}$ in the current round and 0 means that $\mathrm{CH}_{j}$ is not a relay node of any $\mathrm{CH}$ in the current round. $\mathrm{RN}_{-} \mathrm{CH}_{i}$ is defined as denoted in

RN_CH $H_{i}=\min \left\{\mathrm{CH}_{j} \mid \cos t\left(\mathrm{CH}_{i}, \mathrm{CH}_{j}\right), \mathrm{CH}_{j} \in \mathrm{NBr} r_{\mathrm{CH} i}\right\}$.

In general, $\mathrm{CH}_{i}$ can only find one $\mathrm{RN} \mathrm{CH}_{i}$ in a round, which can largely avoid the primary conflict among $\mathrm{CHs}$ in intercluster communication. However, when the $\mathrm{RN}_{-} \mathrm{CH}_{i}$ fails, $\mathrm{CH}$ communicates with the candidate relay node of $\mathrm{CH}_{i}\left(\mathrm{CRN} \mathrm{CH}_{i}\right)$ to ensure the robustness of the IoT. $\mathrm{CRN} \mathrm{CH}_{i}$ is defined as in

$$
C R N_{-} C H_{i}=\min \left\{C H_{j} \mid \cos t\left(C H_{i}, C H_{j}\right), C H_{j} \in N_{B r} r_{i}, C H_{j} \cap R N \_C H_{i}=\varnothing\right\} .
$$

If $\mathrm{CH}_{i}$ cannot find RN_ $\mathrm{CH}_{i}$ and $\mathrm{CRN} \_\mathrm{CH}_{i}$, then the next hop can be found from the FL_CH through formula (2).

4.2. Levels of CHs Based on Transmission Chain. After unequal clustering and multihop routing, the level of the $\mathrm{CH}$ $\left(\mathrm{LCH}_{i}\right)$ is defined as the number of hops $\left(\mathrm{CH}_{i-}\right.$ hop) from $\mathrm{CH}$ to $\mathrm{BS}$ based on the transmission chain.

$$
L C H_{i}=\mathrm{CH}_{i_{-}} \text {hop, }
$$

$$
\begin{aligned}
\text { s.t } & \\
C 1: \forall C H_{i} \in & F L_{-} C H, L C H_{i}=L_{1}, \\
C 2: \forall C H_{n} \in & C H_{L x}, C H_{m} \in C H_{L y}, \text { if } C H_{n-} \text { hop } \\
& <C H_{m-} \text { hop, then } L_{x}<L_{y}, C 3: \\
& C H_{L 1} \cap C H_{L 2} \cap \ldots \cap C H_{L \max }=\phi, \\
C 4: \sum_{i=0}^{\max } N_{-} C_{L i}= & K .
\end{aligned}
$$

Here, the maximum level of $\mathrm{CH}$ is $L_{\max }$ and the number of $\mathrm{CH}$ in the entire network is $\mathrm{K}$. $\mathrm{CH}_{\mathrm{Li}}$ is the set of $\mathrm{CH}$ in $L_{\mathrm{i}}$. The condition $\mathrm{C} 1$ indicates that the level of $\mathrm{CH}$ in $\mathrm{FL} \_\mathrm{CH}$ is $L_{1}$. C2 indicates that the smaller the hop to BS, the smaller the level of $\mathrm{CH}$. $\mathrm{C} 3$ indicates that one $\mathrm{CH}$ cannot belong to different levels at the same time. $\mathrm{C} 4$ indicates that all the $\mathrm{CHs}$ are assigned corresponding levels.

$\mathrm{CHs}$ are layered by BS according to the routing information. The BS broadcasts the number of $\mathrm{CHs}$ in level
(N_CHLi) and the number of CHs in the transmission chain $\left(\mathrm{N}_{-} \mathrm{CHi}_{\mathrm{TC}}\right)$ of all $\mathrm{CH}_{\mathrm{i}}$.

4.3. RSUC Resource Scheduling. With the operation of the network, some nodes will fail, and the topology will be changed dynamically. The joint allocation of channels and time slots adapts to dynamic topology changes. The RSUC scheduling is based on unequal clustering, multihop routing, and the level of $\mathrm{CH}$.

In RSUC, the first channel is used for broadcasting. The number of clusters in the first level and the max number of neighbor clusters within one hop are assumed to not exceed the number of available channels. After the network is initialized or reclustered, the $\mathrm{CH}$ s of the first level and $\mathrm{CH}$ within one hop are assigned different channels, which is based on the graph coloring in a previous study [35]. The channel allocation can avoid the secondary conflicts in intercluster communication. The number of channels ( $\left.N_{\text {channel }}\right)$ used in RSUC is shown in

$N_{\text {Channel }}=\max \left\{N_{-} C H_{L 1}, \max \left\{N_{-} N B r_{C H_{i}}, i-1,2, \ldots, K\right\}\right\}$,

where $\mathrm{N} \_\mathrm{NBr}_{\mathrm{CHi}}$ represents the number of neighbor nodes of $\mathrm{CH}_{\mathrm{i}}$. $\mathrm{K}$ represents the number of $\mathrm{CHs}$ in the entire network.

The transmission slot structure of $\mathrm{CH}$ and $\mathrm{CM}$ in intracluster communication is shown in Figure 4. The time slots of $\mathrm{CH}$ consist of intrabroadcasting slots, intracluster communication slots, handling slots, sleeping slots, 


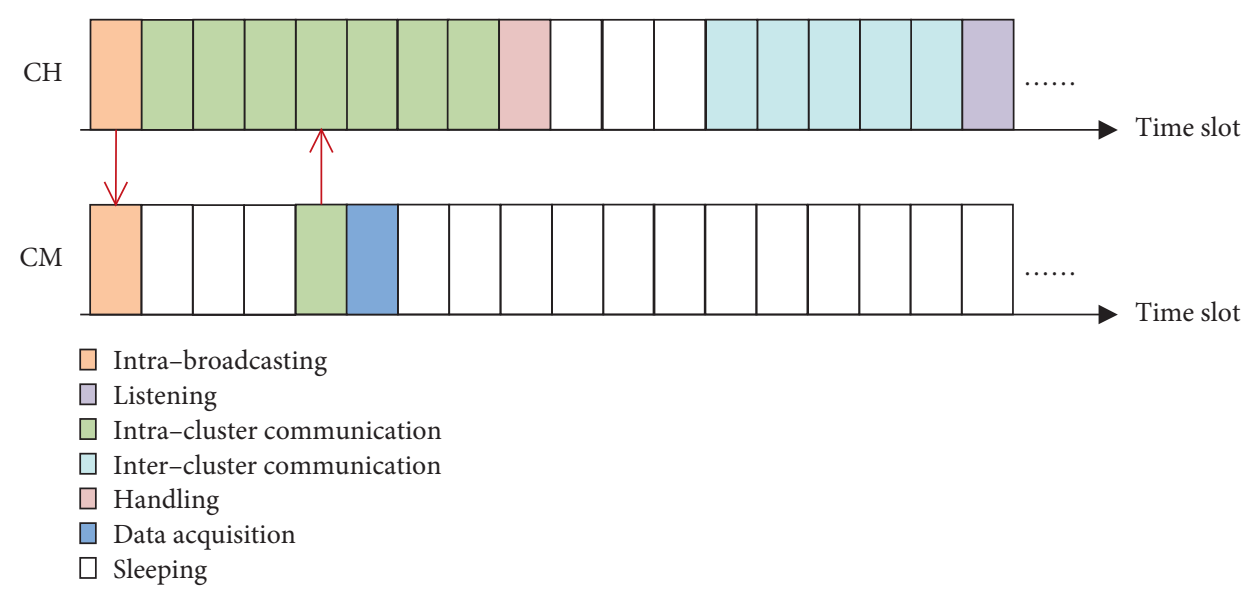

FIgURE 4: Transmission slot structure of intracluster communication.

intercluster communication slots, and listening slots. The time slots of the CM include receiving broadcast slots, intracluster communication slots, data acquisition slot, and sleeping slots.

The transmission slot structure of the $\mathrm{CH}_{\mathrm{Li}}$ and $\mathrm{CH}_{\mathrm{Li}+1}$ in intercluster communication is shown in Figure 5. The number of intracluster communication time slots of $\mathrm{CHs}$ far from the BS is more than the number of those close to the BS, which can be attributed to the unequal clustering. Meanwhile, the number of intercluster communication time slots of CHs far from the BS is less than the number of those far from the BS.

4.3.1. Resource Scheduling of Intracluster Communication. $\mathrm{CH}$ and the $\mathrm{CM}$ thereof use the same channel in intracluster communication. The $\mathrm{CH}$ selects the candidate cluster head $(\mathrm{CCH})$ of the next round that can reduce the energy consumption of reclustering, and the $\mathrm{CCH}$ has the most remaining energy in the cluster.

$$
C C H=\left\{C M_{i} \mid E_{C M i}>T C H_{E n e r g y} \& \& E_{C M i}>E_{C M j}, j=1,2, \ldots, N \_C M-1\right\} .
$$

In intracluster communication, the $\mathrm{CH}$ sends reclustered information to the BS and generates a new TDMA scheduling table when the number of cluster members (N_CM) is less than the threshold of the number of cluster members $\left(T_{\mathrm{N} \_\mathrm{CM}}\right)$. The $\mathrm{CM}$ is invalid when the $\mathrm{CH}$ does not receive data of the CM consecutively T_invalidNum times. If there are failed nodes in the cluster, but the N_CM is not reduced to TN_CM, the CH needs to recycle the resources of the failed nodes and adjust the time slot schedule locally. The $T_{\mathrm{N} \_\mathrm{CM}}$ is defined as follows:

$$
T_{N_{-} C M}=N_{-} C M_{\text {init }}-T M,
$$

where N_CM $\mathrm{CM}_{\text {init }}$ represents the initial number of cluster members and TM represents the determined number according to the actual environment.

The $\mathrm{CH}$ broadcasts information, including intracluster time synchronization, information of $\mathrm{CCH}$, cluster channel, and the TDMA scheduling table. The information of intracluster time synchronization includes the ID of $\mathrm{CH}$, information of time, and the next broadcast time of the $\mathrm{CH}$.

In RSUC, dynamic adaption to topology changes caused by failed nodes and recycle resources of the failed nodes is considered in resource scheduling. The resource scheduling of intracluster communication in RSUC is shown in Figure 6.
$\mathrm{S}_{1}$ : unequal clusters are formed, and clusters in the first level and clusters within the range of one hop are assigned different channels.

$\mathrm{S}_{2}$ : if Round $=1$, the $\mathrm{CH}$ generates the TDMA scheduling table and assigns each CM one-time slot. The CMs are sorted by the ID thereof. The time slots of $\mathrm{CH}$ in intracluster communication $\left(\mathrm{TS}_{\mathrm{CH} \text {-intra }}\right)$ are defined as denoted in

$$
T S_{C H-\text { intra }}=\sum_{i=1}^{N_{-} C M} N D_{-} C M_{i} \times T_{\text {unit }} .
$$

where ND_CM represents the amount of data in the cluster member. $\mathrm{T}_{\text {unit }}$ represents the time required to send one unit of data.

The time slots of $\mathrm{CM}_{\mathrm{i}}\left(\mathrm{TS}_{\mathrm{CMi}}\right)$ are defined as denoted in

$$
T S_{C M i}=N D_{-} C M_{i} \times T_{\text {unit }} .
$$

$\mathrm{S}_{3}$ : the CM transmits data and the remaining energy thereof to $\mathrm{CH}$ in the corresponding time slot. The $\mathrm{CH}$ receives the data of $\mathrm{CM}$ in the time slot of intracluster communication and performs data fusion and handling. If $\mathrm{CH}$ does not receive data of $\mathrm{CM}$ consecutively T_invalidNum times, the CM is determined to be invalid, 


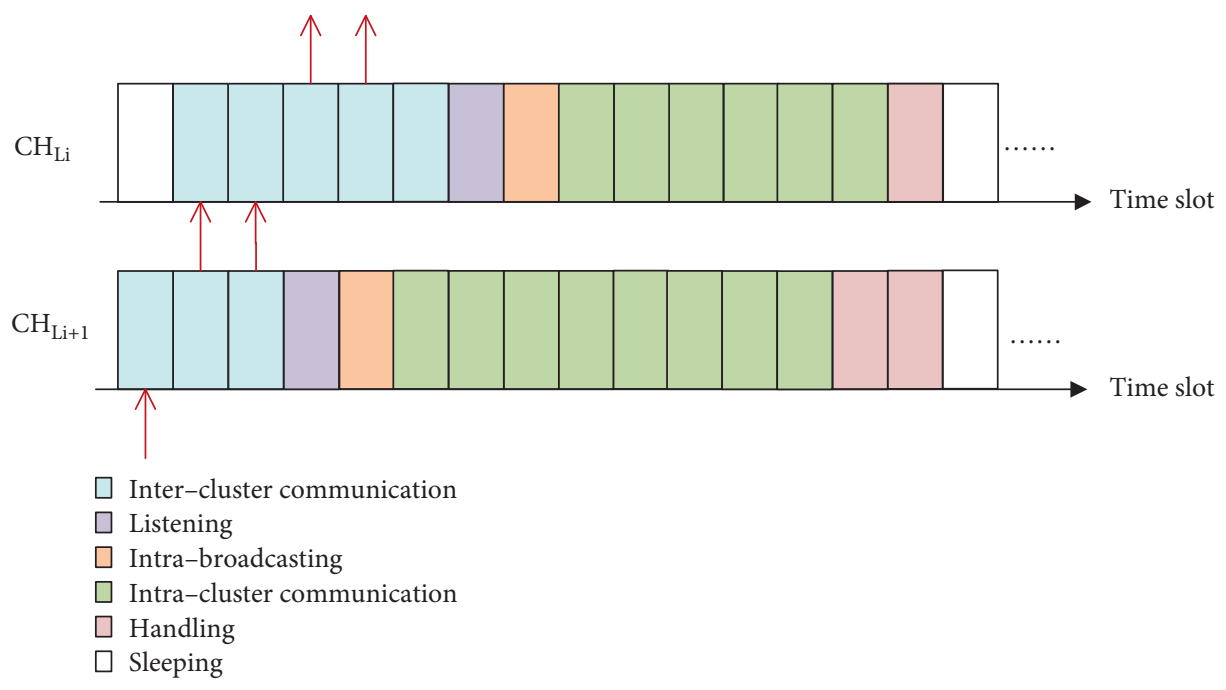

FIGURE 5: Transmission slot structure of intercluster communication.

and N_CM $=$ N_CM-1. If the current round does not end, $\mathrm{S}_{3}$ is skipped to; otherwise, $\mathrm{S}_{4}$ can be proceeded to.

$\mathrm{S}_{4}$ : Round $\leftarrow$ Round +1 . The $\mathrm{CCH}$ becomes the new $\mathrm{CH}$ in the cluster.

$\mathrm{S}_{5}$ : if there are no failed nodes in the cluster, the nodes in the cluster partly reuse the previous round of the scheduling table. The previous round of $\mathrm{CH}$ becomes the normal CM and uses the time slot of the $\mathrm{CCH}$ in the previous round. The scheduling order of other nodes remains the same. The starting points of the time slots are added with corresponding time differences, and S3 is skipped to; otherwise, S6 can be proceeded to.

$\mathrm{S}_{6}$ : if there are failed nodes in the cluster, but the number of CMs in the cluster is not reduced to the threshold $T_{\mathrm{N} \_\mathrm{CM}}$, the $\mathrm{CH}$ needs to recycle the resources of the failed nodes and adjust the time slot schedule locally. The time slots of nodes behind failed nodes are subtracted accordingly, and S3 is skipped to. The failed nodes are handled internally by $\mathrm{CH}$ without notifying BS so as to reduce the impact of failed nodes on the whole network; otherwise, $\mathrm{S}_{7}$ can be proceeded to.

S7: if the number of CMs in the cluster is reduced to the threshold TN_CM, the CH notifies the BS to recluster and generate a new TDMA scheduling table.

The steps of resource scheduling of intracluster communication in RSUC are as follows:

\subsubsection{Resource Scheduling of Intercluster Communication.} Due to the data among clusters potentially being highly different, data fusion in intercluster communication is not performed. The $\mathrm{CH}$ switches to the channel of the relay node thereof when entering intercluster communication. In RSUC, multiple CHs can communicate in parallel via effective joint allocation of time slots and channels.

The time slot allocation of intercluster communication is based on channel allocation. In intercluster communication, $\mathrm{CH}$ has two different states, sending state $(\mathrm{S})$ and receiving state (R). CH can only be in one state at the same time. The state transition of the $\mathrm{CH}$ consumes part of the energy. In order to save the energy consumption of the transition state, aside from the first time slot, the rest are allocated two consecutive time slots in each round in RSUC. The resource scheduling for intercluster communication in RSUC is shown in Figure 7.

The steps of resource scheduling of intercluster communication in RSUC are as follows:

$\mathrm{S}_{1}$ : the adjacent $\mathrm{CHs}$ are assigned different channels by using the coloring algorithm. For each transmission chain, if the number of $\mathrm{CHs}$ in the transmission chain $\left(\mathrm{N}_{-} \mathrm{CHi}_{\mathrm{TC}}\right)$ is odd, the CHs in odd levels are in a state of $\mathrm{S}$, and the $\mathrm{CHs}$ in even levels are in a state of $\mathrm{R}$; otherwise, the states of $\mathrm{CH}$ in even levels are $\mathrm{S}$ and the states of CHs in odd levels are R.

$\mathrm{S}_{2}$ : the $\mathrm{CHs}$ at $\mathrm{S}$ state switch their channel to the corresponding relay node $(\mathrm{RN})$.

$\mathrm{S}_{3}$ : during transmission in the first round, one sending time slot is allocated to all the $\mathrm{CH}$ in the S state, and one receiving time slot is allocated to all the $\mathrm{CHs}$ in the $\mathrm{R}$ state. The time slot is $1, \mathrm{TS}_{1}=1$.

$\mathrm{S}_{4}$ : the $\mathrm{CHs}$ in $\mathrm{S}$ state send data to the corresponding $\mathrm{RN}$, which is in state $\mathrm{R}$. If $\mathrm{CH}_{\mathrm{i}}$ is the last-of-chain node of the transmission chain, the information of the end of data in $\mathrm{CH}_{\mathrm{i}}\left(\mathrm{EOD} \_\mathrm{CH}_{\mathrm{i}}\right)$ is carried. After transmitting the data, the $\mathrm{CH}$ enters a listening time slot.

$\mathrm{S}_{5}$ : after the first round, $\mathrm{TS}_{2}=1$ is initialized. There can be a protection time slot in the middle of the allocated time slots.

$\mathrm{S}_{6}$ : aside from $\mathrm{CHs}$ that have transmitted EOD_CH, all the other $\mathrm{CH}$ s change their state; namely, the $\mathrm{CH}$ in $\mathrm{S}$ state is changed to $\mathrm{R}$, and $\mathrm{CH}$ in $\mathrm{R}$ state is changed to $\mathrm{S}$.

$\mathrm{S}_{7}$ : two consecutive sending time slots are assigned to each $\mathrm{CH}$ at $\mathrm{S}$, and two consecutive receiving time slots are assigned to each $\mathrm{CH}$ at $\mathrm{R}$. $\mathrm{CH}$ is allocated to two consecutive time slots $\mathrm{TS}_{1}$ and $\mathrm{TS}_{2}$, respectively, 


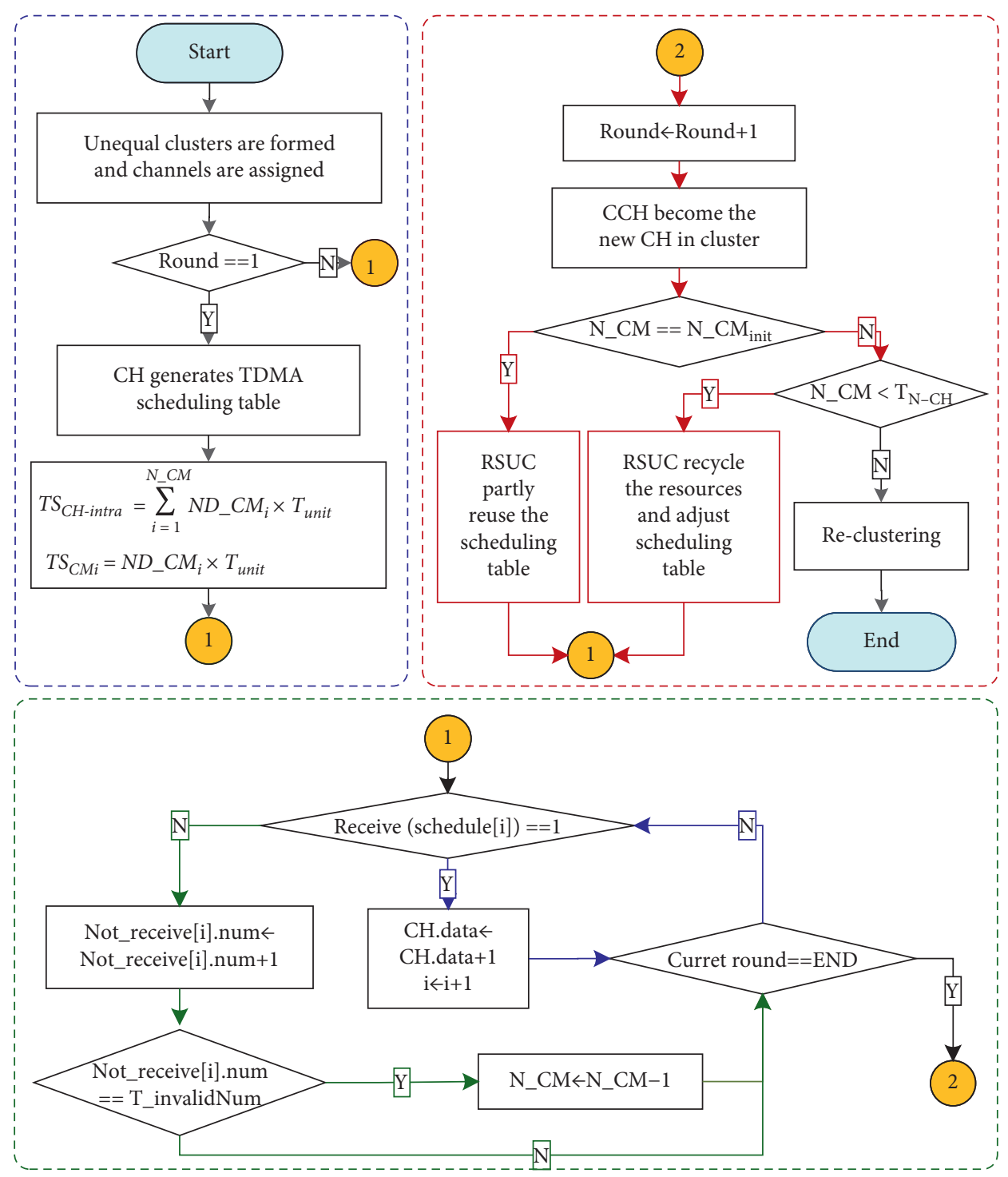

FIgURE 6: Resource scheduling of intracluster communication.

$\mathrm{TS}_{1}=\mathrm{TS}_{2}++$ and $\mathrm{TS}_{2}=\mathrm{TS}_{1}++$. For example, in the second round, $\mathrm{TS}_{1}=2$ and $\mathrm{TS}_{2}=3$. In the third round, $\mathrm{TS}_{1}=4$ and $\mathrm{TS}_{2}=5$. In the fourth round, $\mathrm{TS}_{1}=6$ and $\mathrm{TS}_{2}=7$.

$\mathrm{S}_{8}$ : if the $\mathrm{CH}_{\mathrm{j}}$ receives the data identified by $\mathrm{EOD} \_\mathrm{CH}_{\mathrm{i}}$, the $\mathrm{CH}_{\mathrm{j}}$ sends data carrying EOD_CH $\mathrm{CH}_{\mathrm{j}}$, which may include data of the $\mathrm{CH}_{\mathrm{j}}$. After the $\mathrm{CH}$ transmits the data carrying EOD_CH, the $\mathrm{CH}$ enters the listening time slot. If no other data is received in the listening time slot, the $\mathrm{CH}$ enters intracluster communication.

$\mathrm{S}_{9}$ : if all the $\mathrm{CHs}$ enter intracluster communication, the scheduling algorithm is stopped; otherwise, $\mathrm{S}_{6} \sim \mathrm{S}_{9}$ are looped until all the $\mathrm{CHs}$ enter intracluster communication.

Compared with the PIP algorithm, in RSUC, the amounts of state transition and channel switching are reduced by about half because two consecutive time slots are assigned.
If $\mathrm{CH}_{\mathrm{i}}$ finds that the $\mathrm{RN} \_\mathrm{CH}_{\mathrm{i}}$ has failed during transmission, the $\mathrm{CH}$ will buffer the received data. The $\mathrm{CH}$ of the lower level and upper level of the $\mathrm{RN} \mathrm{CH}_{\mathrm{i}}$ will transmit data as usual. The $\mathrm{CH}$ sends the data in the buffer thereof to the CRN_CH $\mathrm{CH}_{\mathrm{i}}$ using consecutive time slots until the listening time slot of CRN_CH . The CRN_CH $\mathrm{CH}_{\mathrm{i}}$ sends the data to the $\mathrm{RN}$ with consecutive time slots in the listening time slot until the data finally arrive at the BS.

An example of joint allocation of channel and time slots for intercluster communication in RSUC is shown in Figure 8 . To send all data to the BS in one round, 6 channels and 5-time slots are utilized. $C_{\mathrm{i}}$ indicates that $\mathrm{CH}$ uses channel $i$, and $\mathrm{TS}_{\mathrm{j}}$ indicates that $\mathrm{CH}$ is allocated time slot $j$. Generally, the $\mathrm{CH}$ s at the lower level complete the intercluster communication earlier than the $\mathrm{CH}$ s at the upper level. The number of intercluster communication time slots of the $\mathrm{CH}$ near the BS is more than the number of those far from the BS, which can be attributed to the unequal clustering topology. The number of intracluster communication time 


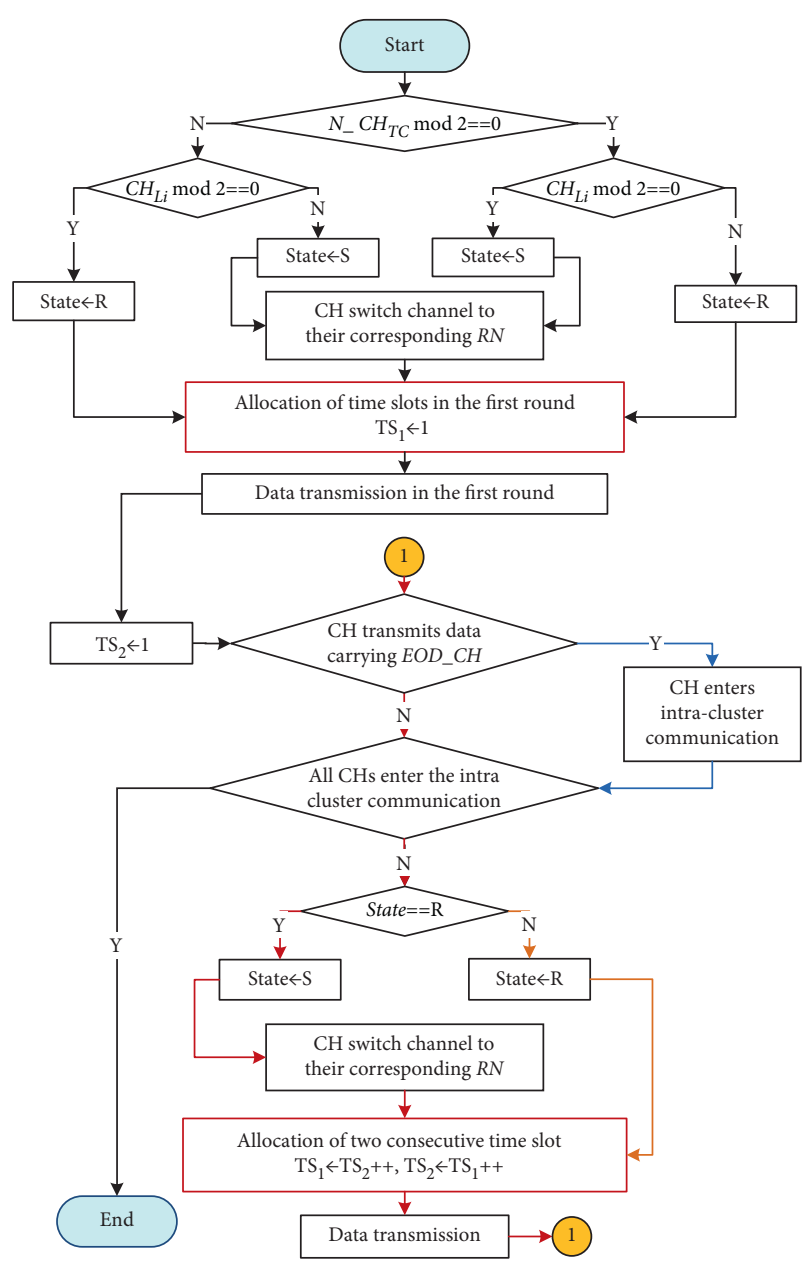

FIGURE 7: Resource scheduling of intercluster communication.

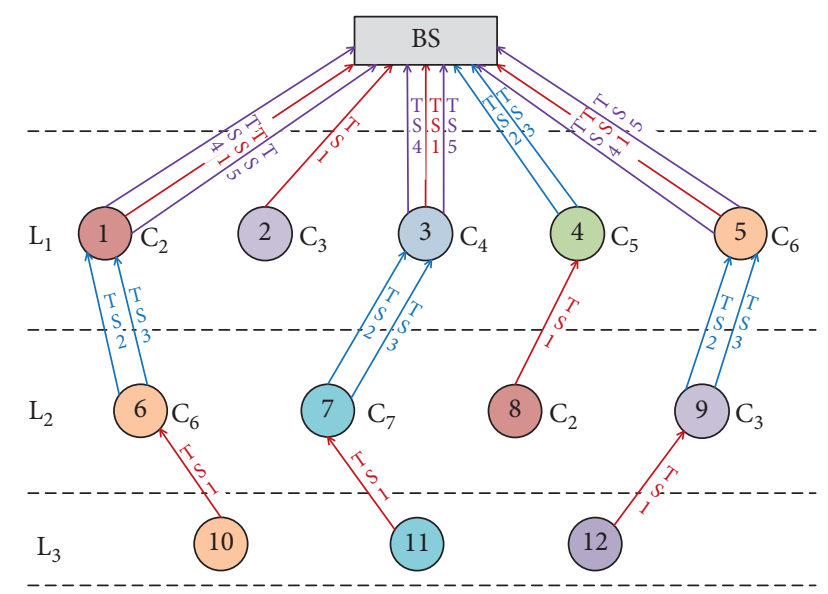

FIGURE 8: Joint allocations of channels and time slots in intercluster communication.

slots of $\mathrm{CH}$ far from the $\mathrm{BS}$ is more than the number of those close to the BS. In RSUC, an attempt is made to maximize concurrent transfers over each time slot based on unequal clustering.
Figure 9 shows the results of joint allocation of intercluster communication in RSUC. S indicates that the $\mathrm{CH}$ is in the sending state. $\mathrm{R}$ indicates that the $\mathrm{CH}$ is in the receiving state. $E$ indicates that the $\mathrm{CH}$ transmitted data with EOD_CH in Slot ${ }_{i}$. Figure 9 shows the corresponding time slots and channels of each node, as well as the levels and corresponding states of the node.

The overall operation process of RSUC resource scheduling is shown in Figure 10.

\section{Theoretical Analysis}

Theorem 1. The RSUC resource scheduling is interferencefree.

Proof. In RSUC, the data transmission is divided into intracluster communication and intercluster communication:

(1) In intracluster communication, the CMs communicate with the $\mathrm{CH}$ thereof in one hop in the cluster channel thereof. Adjacent clusters work in different channels. Thus, there are no secondary conflicts in a cluster. As intracluster communication is based on TDMA scheduling, primary conflicts are eliminated.

(2) In intercluster communication, all the $\mathrm{CHs}$ within one hop use different channels. The $\mathrm{CH}$ switches to the channel of the $\mathrm{RN}$ thereof, thereby removing secondary conflicts. CHs in odd levels and even levels use different sending time slots. As $\mathrm{CHs}$ only communicate with one $\mathrm{RN}$ at one time, primary conflicts between clusters are eliminated.

Therefore, there are no primary and secondary conflicts for any links in data transmission. RSUC is interferencefree.

Theorem 2. In RSUC, for any $\mathrm{CH}_{i}$ in transmission chain ${ }_{j}$, intercluster communication is completed in TS_CH $\mathrm{H}_{i}$ time slots when there are no failed CHs. TS_CH ${ }_{i}$ is shown in the following formula:

$$
T S_{-} C H_{i}=2 \times\left(N_{-} C_{i T C}-L C H_{i}\right)+1,
$$

where $\mathrm{N}_{-} \mathrm{CH}_{T C}$ represents the number of $\mathrm{CHs}$ in the transmission chain of $\mathrm{CH}_{i}$. $\mathrm{LCH}_{i}$ represents the level of $\mathrm{CH}_{i}$.

Proof. If $\mathrm{CH}_{\mathrm{M}}$ is in the bottom level of the transmission chain ${ }_{j}, \mathrm{CH}_{\mathrm{M}}$ transmits the data to the $\mathrm{RN} \_\mathrm{CH}_{\mathrm{M}}$ thereof, in which the used time slot is 1 . Namely, $\mathrm{TS}_{-} \mathrm{CH}_{\mathrm{M}}=1$.

If $\mathrm{CH}_{\mathrm{i}}=\mathrm{CH}_{\mathrm{M}-1}$ (the second bottom layer), the $\mathrm{CH}_{\mathrm{i}}$ receives the data of the $\mathrm{CH}_{M}$ in time slot 1 . In time slots 2 and 3 , the $\mathrm{CH}_{\mathrm{i}}$ sends the data thereof and the data of $\mathrm{CH}_{\mathrm{M}}$ to RN_CH $\mathrm{CH}_{\mathrm{M}-1}$. Thus, TS_CH ${ }_{\mathrm{M}-1}=3$.

If $\mathrm{CH}_{\mathrm{i}}=\mathrm{CH}_{\mathrm{M}-2}$, the $\mathrm{CH}_{\mathrm{i}}$ sends the data thereof in time slot 1. In time slots 2 and 3, the data of $\mathrm{CH}_{\mathrm{M}-1}$ is received. In time slots 4 and 5 , the $\mathrm{CH}_{\mathrm{i}}$ sends data of $\mathrm{CH}_{\mathrm{M}-1}$ to RN_ $\mathrm{CH}_{\mathrm{M}-2}$. Thus, TS_CH ${ }_{\mathrm{M}-2}=5$. 


\begin{tabular}{|c|c|c|c|c|c|}
\hline Nodes & Slot1 & Slot2 & Slot3 & Slot4 & Slot5 \\
\hline BS & $4 \mathrm{R}$ & $\mathrm{R}$ & $\mathrm{R}$ & $3 \mathrm{R}$ & $3 \mathrm{R}$ \\
\hline 1 & $\mathrm{~S}$ & $\mathrm{R}$ & $\mathrm{R}$ & $\mathrm{S}$ & $\mathrm{S}$ \\
\hline 2 & $\mathrm{~S}$ & $\mathrm{E}$ & & & \\
\hline 3 & $\mathrm{~S}$ & $\mathrm{R}$ & $\mathrm{R}$ & $\mathrm{S}$ & $\mathrm{S}$ \\
\hline 4 & $\mathrm{R}$ & $\mathrm{S}$ & $\mathrm{S}$ & $\mathrm{E}$ & \\
\hline 5 & $\mathrm{~S}$ & $\mathrm{R}$ & $\mathrm{R}$ & $\mathrm{S}$ & $\mathrm{S}$ \\
\hline 6 & $\mathrm{R}$ & $\mathrm{S}$ & $\mathrm{S}$ & $\mathrm{E}$ & \\
\hline 7 & $\mathrm{R}$ & $\mathrm{S}$ & $\mathrm{S}$ & $\mathrm{E}$ & \\
\hline 8 & $\mathrm{~S}$ & $\mathrm{E}$ & & & \\
\hline 9 & $\mathrm{R}$ & $\mathrm{S}$ & $\mathrm{S}$ & $\mathrm{E}$ & \\
\hline 10 & $\mathrm{~S}$ & $\mathrm{E}$ & & & \\
\hline 11 & $\mathrm{~S}$ & $\mathrm{E}$ & & & \\
\hline 12 & $\mathrm{~S}$ & $\mathrm{E}$ & & & \\
\hline
\end{tabular}

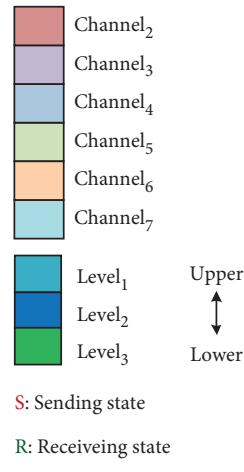

E: End of Data

FIGURE 9: Results of joint allocation of intercluster communication.

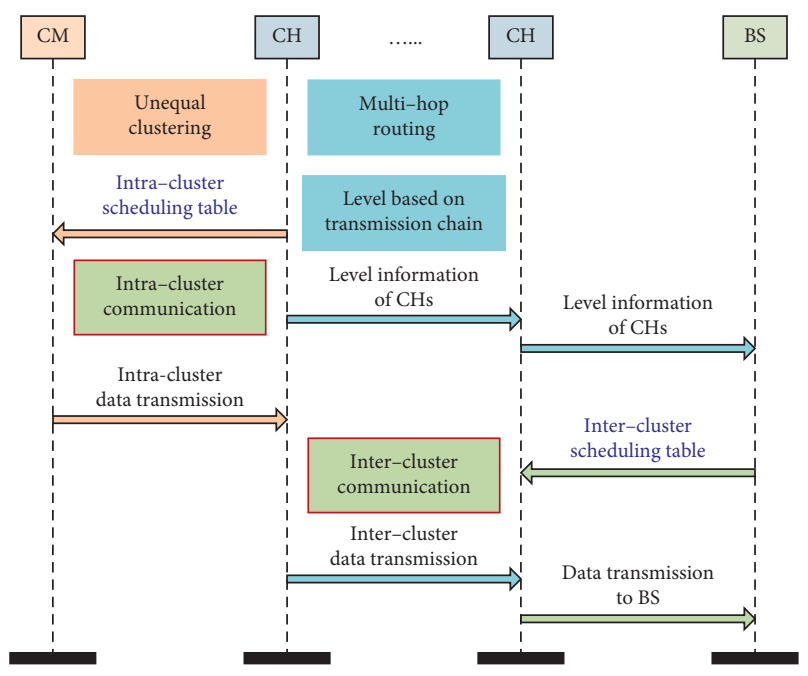

FIgURE 10: Operation process of RSUC.

By analogy,

$$
T S \_C H_{i+1}=T S \_C H_{i}-2 .
$$
then

If $\mathrm{CH}_{\mathrm{i}}$ and $\mathrm{CH}_{\mathrm{i}+1}$ are in the same transmission chain,

$$
L C H_{i}=L C H_{i+1}-1 .
$$

If $\mathrm{CH}_{\mathrm{i}}$ is in the bottom level of the transmission chain ${ }_{\mathrm{j}}$, then $\mathrm{LCH}_{\mathrm{i}}=\mathrm{N}_{-} \mathrm{CHi}_{\mathrm{TC}}$. By combining with formula (12),

TS_CH $H_{i}=2 \times\left(N_{-} C_{i_{T C}}-L C H_{i}\right)+1=2 \times 0+1=1$.

Thus, formula (12) is established when $\mathrm{CH}_{\mathrm{i}}$ is at the bottom level.

An assumption is made that for any $\mathrm{CH}_{\mathrm{i}}$ in the transmission chain ${ }_{\mathrm{j}}$, TS_CH $\mathrm{CH}_{\mathrm{i}}=2 \times\left(\mathrm{N}_{-} \mathrm{CHi}_{\mathrm{TC}}-\mathrm{LCH}_{\mathrm{i}}\right)+1$ is established. According to formulas (13) and (14), the TS_CH $\mathrm{CH}_{\mathrm{i}+1}$ is shown as follows: TS_CH $\mathrm{CH}_{\mathrm{i}+1}=\mathrm{TS}_{-} \mathrm{CH}_{\mathrm{i}}-2=(2 \times$ $\left.\left(\mathrm{N}_{-} \mathrm{CHi}_{\mathrm{TC}}-\mathrm{LCH}_{\mathrm{i}}\right)+1\right)-2=\left(2 \times\left(\mathrm{N}_{-} \mathrm{CHi}_{\mathrm{TC}}-\left(\mathrm{LCH}_{\mathrm{i}+1}\right.\right.\right.$ $-1))+1)-2=2 \times\left(\mathrm{N}_{-} \mathrm{CHi}_{\mathrm{TC}}-\mathrm{LCH}_{\mathrm{i}+1}\right)+1$.

$\mathrm{CH}_{\mathrm{i}}$ and $\mathrm{CH}_{\mathrm{i}+1}$ are in the same transmission chain. Thus, the number of $\mathrm{CHs}$ in the transmission chain is equal. Namely, $\mathrm{N}_{-} \mathrm{CHi}_{\mathrm{TC}}=N_{-} \mathrm{CH}(i+1)_{\mathrm{TC}}$. Then,

$$
T S_{-} C_{i+1}=2 \times\left(N_{-} C H(i+1)_{T C}-L C H_{i+1}\right)+1 \text {. }
$$

By induction, $\mathrm{CH}_{\mathrm{i}}$ in the transmission chain ${ }_{\mathrm{j}}$ completes intercluster communication in $2 \times\left(\mathrm{N}_{-} \mathrm{CHi}_{\mathrm{TC}}-\mathrm{LCH}_{\mathrm{i}}\right)+1$ time slots when there are no failed $\mathrm{CH}$ in RSUC.

Theorem 3. In RSUC, all nodes send data to the BS within TTS time slots in intercluster communication when there are no failed CHs. TTS is shown in the following formula:

$$
T T S=2 \times L_{\max }-1,
$$

where $L_{\max }$ represents the highest level of the longest transmission chain.

Proof. In RSUC, all the data are always forwarded to the BS through the $\mathrm{CH}$ of the first level. The total time slot is directly determined by the $\mathrm{CH}$ of the first level in the longest transmission chain.

Bring $\mathrm{N}_{-} \mathrm{CHi}_{\mathrm{TC}}=L_{\max }$ and $\mathrm{LCH}_{\mathrm{i}}=1$ into formula (12) in Theorem 2 to obtain

$$
\begin{aligned}
\text { TTS } & =2 \times\left(N_{-} C_{i_{T C}}-L_{C H}\right)+1 \\
& =2 \times\left(L_{\max }-1\right)+1 \\
& =2 \times L_{\max }-1 .
\end{aligned}
$$

As such, all nodes send data to the BS within $2 \times L_{\max }-1$ time slots in intercluster communication in the case of no failed CHs.

Through Theorems 2 and 3, an observation can be made that time slots of intercluster communication in RSUC are affected by the level of $\mathrm{CH}$ and the routing transmission chain.

\section{Experiments}

The experiments were perfumed using MATLAB. The simulation parameters are listed in Table 2. IoT composed of 50 300 sensor nodes and a base station was placed at the outside of the sensor field.

Figure 11 shows examples of the unequal clustering and multihop routing in RSUC, in which the network was composed of 300 nodes and $R_{\mathrm{c}}{ }^{0}=40 . R_{\mathrm{c}}{ }^{0}$ represents the initial maximum competition radius. The cost function of the node in multihop routing is defined in formula (2).

Figure 12 shows the average number of clusters at different levels in RSUC. As the layers increased, the number of clusters decreased because of unequal clustering. The present experimental data were obtained on average through hundreds of experimental results. The initial number of sensor nodes and the initial maximum competition radius of unequal clustering affected the number of clusters at different levels.

Figure 13 shows the average number of channels at a different number of nodes. The number of channels used in RSUC was based on formula (7). The channels used in RSUC increased with the number of nodes and decreased with the initial maximum competition radius of unequal clustering. 
TABLE 2: Simulation parameters.

\begin{tabular}{lc}
\hline Parameter & Value \\
\hline Deployment field & $200 \mathrm{~m} \times 200 \mathrm{~m}$ \\
Number of nodes & $50 \sim 300$ \\
Deployment method & Random \\
Initial energy & $50 \mathrm{~J}$ \\
BS position & $(100 \mathrm{~m}, 300 \mathrm{~m})$ \\
Eelce & $50 \mathrm{~nJ} / \mathrm{bit}$ \\
$\square$ fs & $10 \mathrm{pJ} / \mathrm{bit} / \mathrm{m} 2$ \\
$\square \mathrm{mp}$ & $0.0013 \mathrm{pJ} / \mathrm{bit} / \mathrm{m} 4$ \\
EDA & $5 \mathrm{~nJ} / \mathrm{bit} / \mathrm{signal}$ \\
Unit energy of state transformation & $2 \mathrm{~nJ}$ \\
Unit energy of channel switching & $2 \mathrm{~nJ}$ \\
Max data packet size in intracluster & $32 \mathrm{bytes}$ \\
Max data packet size in intercluster & $125 \mathrm{bytes}$ \\
Max control packet size & $8 \mathrm{bytes}$ \\
Total number of channels & 16 \\
Max hop number & Variable \\
Transmission power & Variable \\
T_invalidNum & 3 \\
TM & round(N_CMinit/4) \\
\hline
\end{tabular}

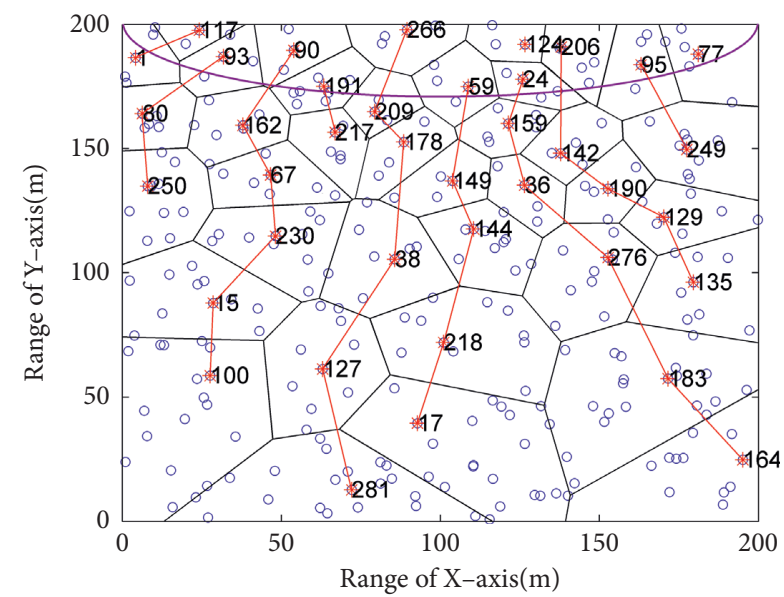

FIgURE 11: Routing based on unequal clustering $\left(N_{\text {int }}=300\right.$; $\left.R_{c}^{0}=40\right)$.

Figure 14 shows the average time slots of intracluster communication at different levels. The number of slots in the intracluster communication was related to the number of cluster members in the cluster. The farther away from the BS, the more the intracluster communication slots in the cluster. As the layers increased, the intracluster communication time slot increased. The resources of failed nodes were dynamically recycled within the range of the threshold, and the previous TDMA scheduling table was partially reused. Thus, the energy of reallocating time slots in intracluster communication of RSUC was saved.

Figure 15 shows the average number of intercluster communication time slots in different levels. As the layers increased, the intercluster communication time slots decreased because of unequal clustering. In RSUC, the $\mathrm{CHs}$ near the BS had more intercluster communication time slots than $\mathrm{CHs}$ far away from BS.

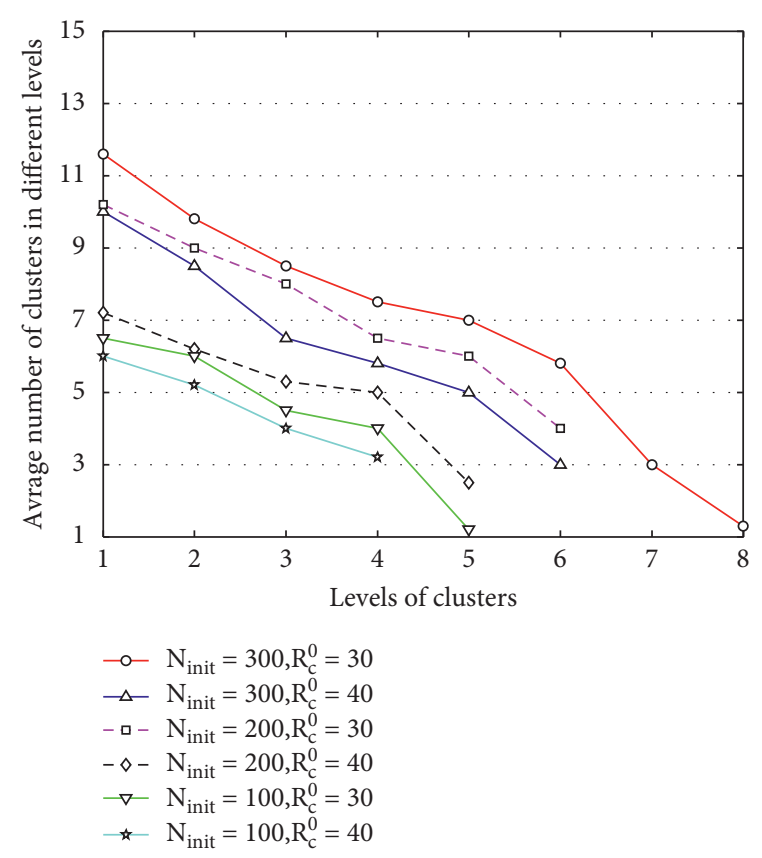

FIGURE 12: Average number of clusters at different levels in RSUC.

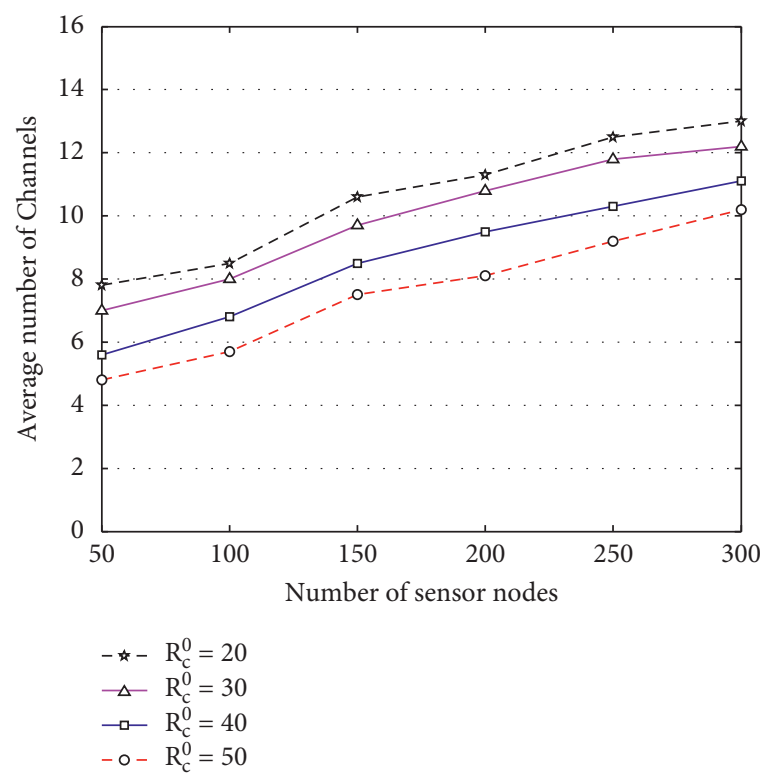

Figure 13: Average number of channels in RSUC.

Figure 16 shows the average max time slots of intercluster communication. The maximum number of time slots for intercluster communication was related to the length of the network routing transmission chain. As the number of sensor nodes increased, the time slots of intercluster communication increased. As $R_{c}^{0}$ increased, the time slots of intercluster communication decreased.

Figure 17 shows the number of state transitions in a round of RSUC and PIP. In PIP, one slot is allocated each time with the status transition and the channel conversion. In RSUC, aside from the first round, the rest are allocated 


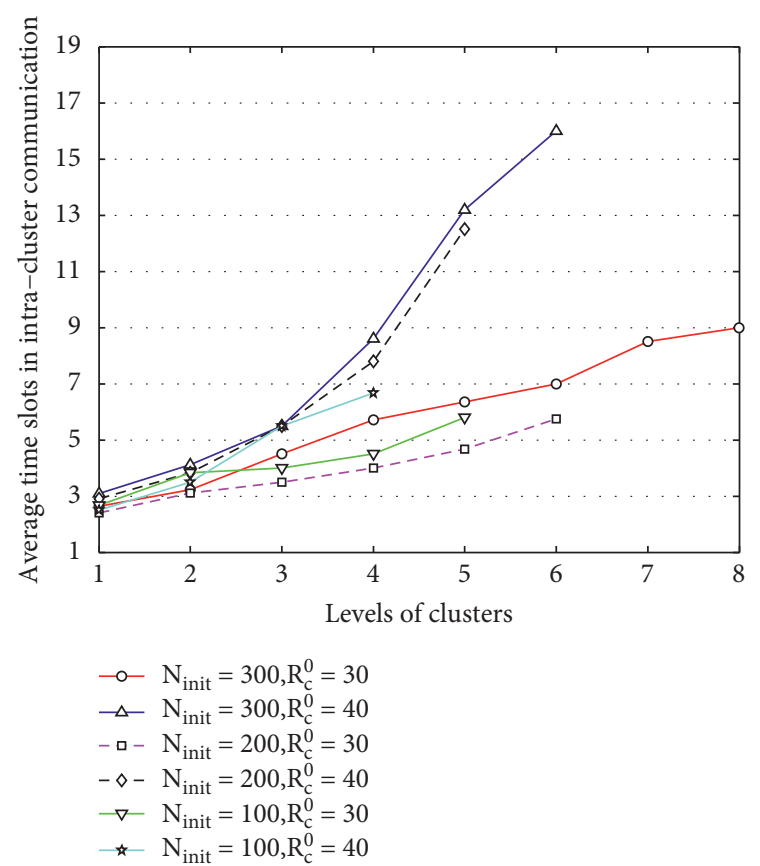

Figure 14: Average time slots in intracluster communication at different levels in RSUC.

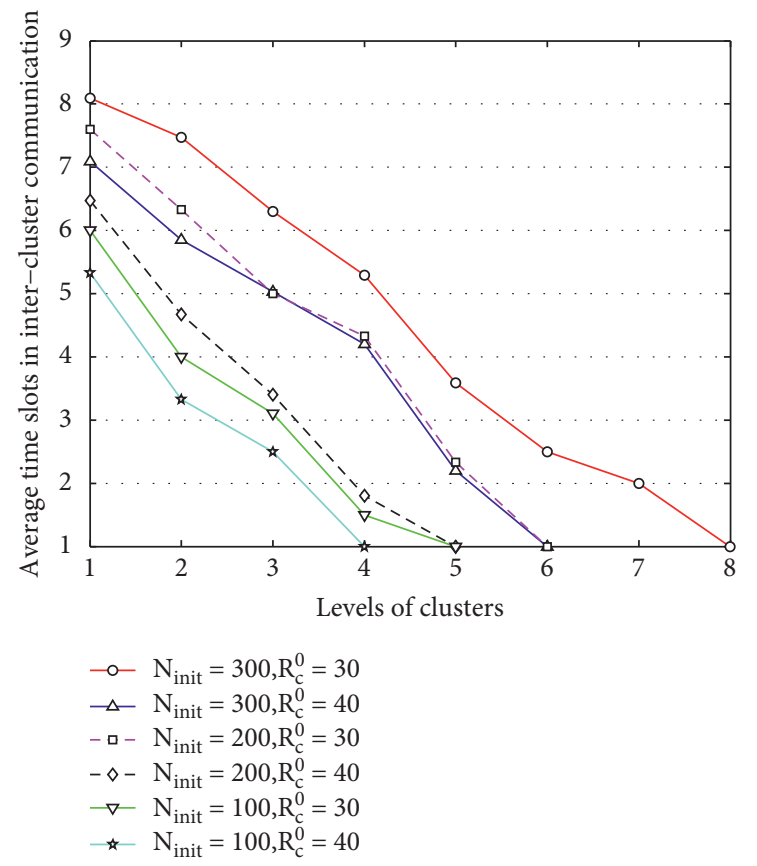

FIGURE 15: Average time slots in intercluster communication at different levels in RSUC.

two consecutive time slots in each round. RSUC reduced the total number of states transition and channel switching by about 45\% compared with PIP.

Figure 18 shows the energy of state transitions and channel switching in a round of intercluster communication. In resource scheduling of intercluster communication in RSUC, the energy consumption of state transitions and

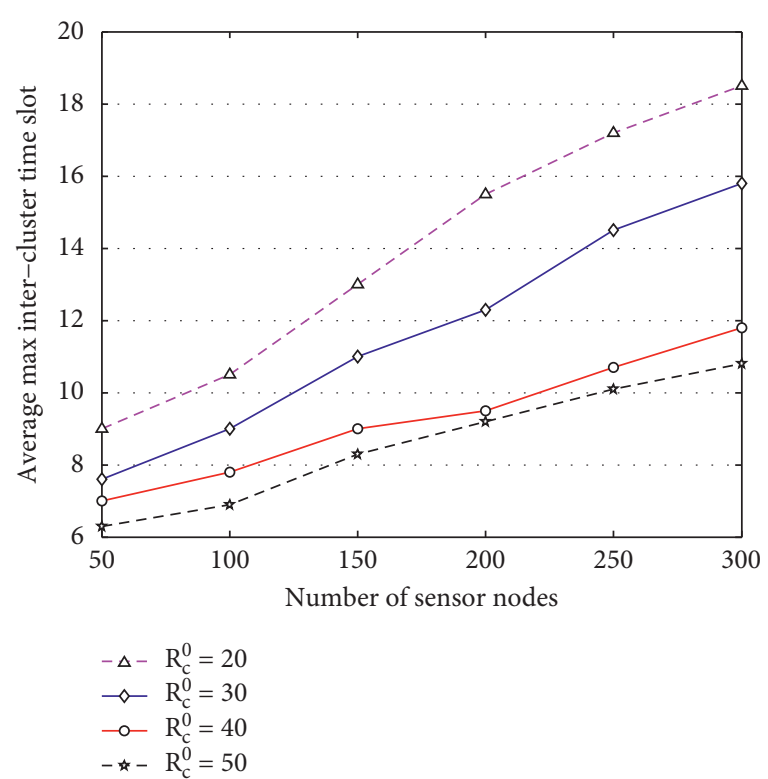

Figure 16: Average max time slots in intercluster communication.

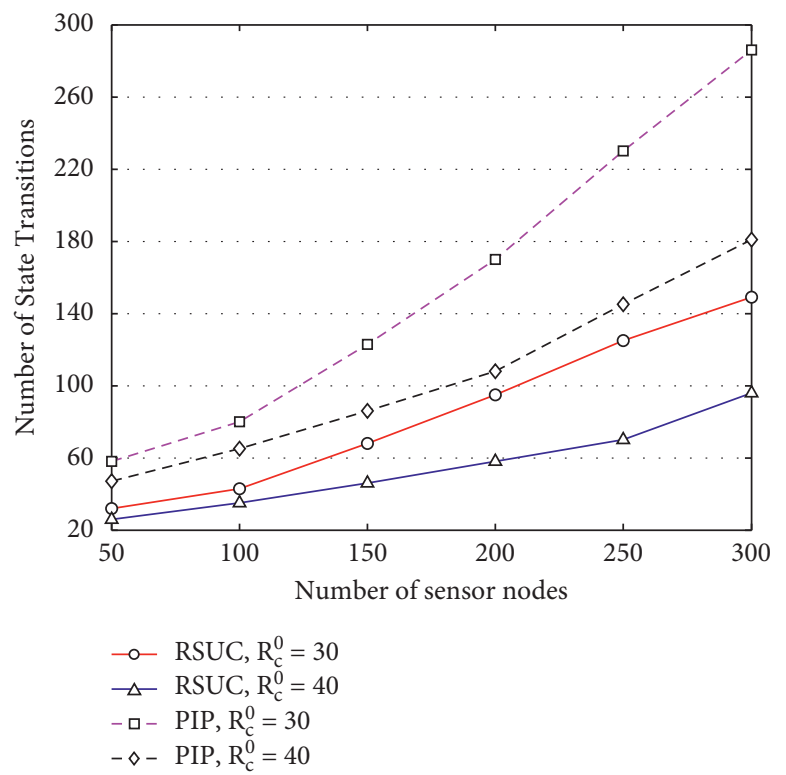

FIGURE 17: Number of state transitions in a round of RSUC and PIP.

channel switching was reduced. Therefore, the RSUC has an advantage in saving energy of transition. Compared with PIP, RSUC reduced the energy of state transitions and channel switching by about $41 \%$.

Figure 19 shows the network throughput of intercluster communication. In RSUC, clusters that are further away from the BS end intercluster communication earlier and enter intracluster communication instead of waiting for all $\mathrm{CHs}$ to complete intercluster communication. Such a method significantly increases the network throughput by effective utilization of time slots. Compared with waiting for all the $\mathrm{CHs}$ to complete intercluster communication before 


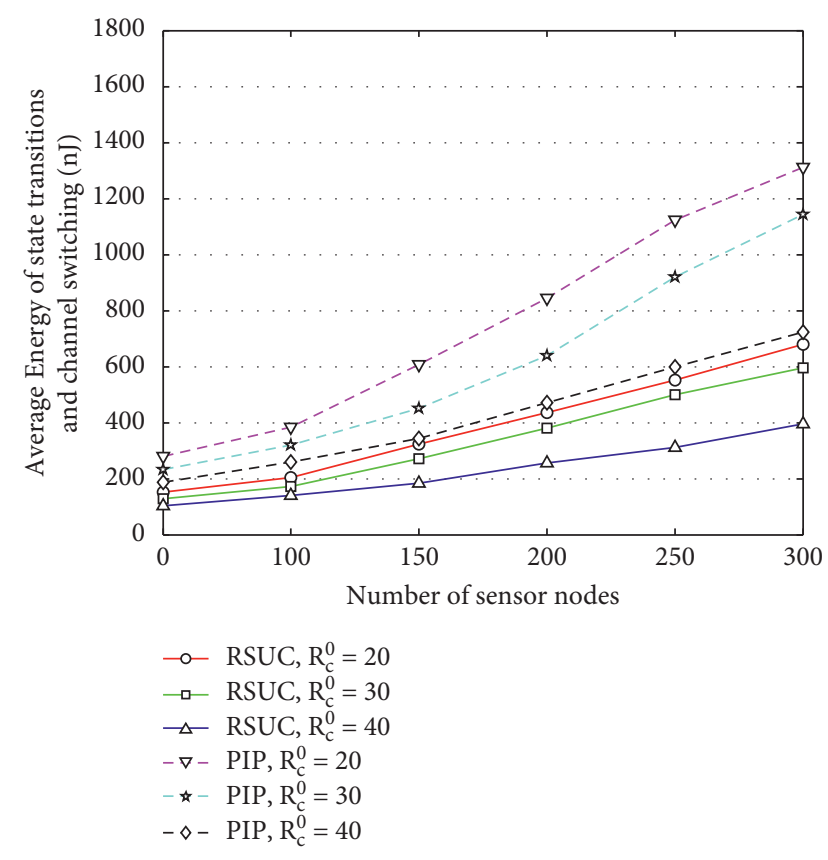

FIGURE 18: Average energy of state transitions and channel switching in a round of intercluster communication of RSUC and PIP.

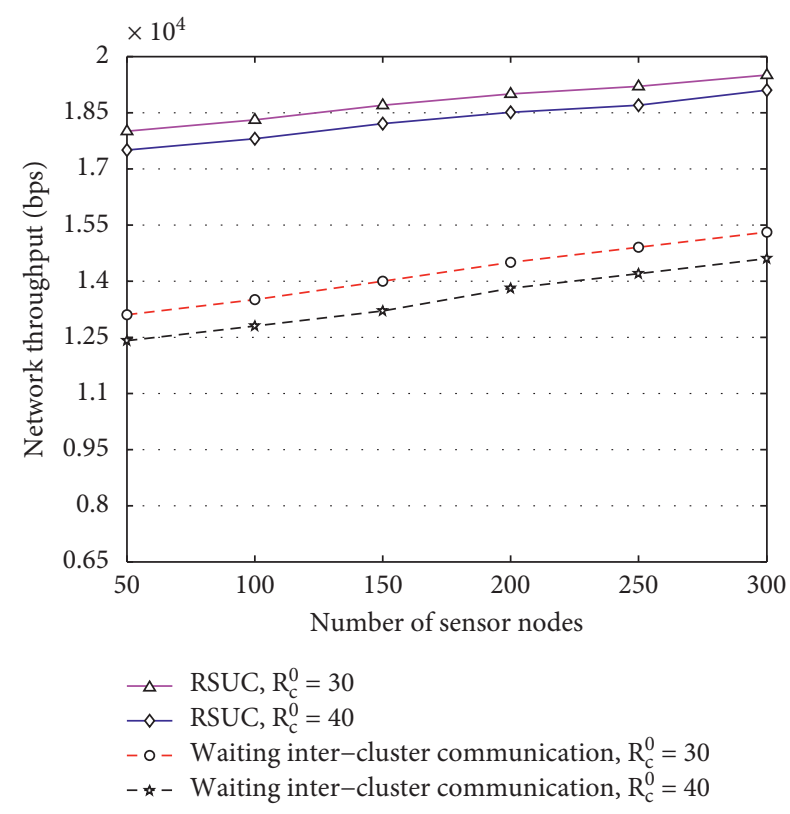

Figure 19: Network throughput.

entering intracluster communication, RSUC increase the network throughput by about $30 \%$.

\section{Conclusion}

Resource scheduling based on unequal clustering in IoT (RSUC) is proposed in the present study, which comprehensively considers the dynamic changes of unequal clustering and multihop routing. In resource scheduling of intracluster communication, the cluster heads recycle resources of failed nodes. In resource scheduling of intracluster communication, multiple $\mathrm{CH}$ s can communicate in parallel via effective joint allocation of time slots and channels. Clusters that are further away from the BS end intercluster communication earlier and enter into intracluster communication instead of waiting for all $\mathrm{CHs}$ to complete intercluster communication. The simulation results show that RSUC reduced the total number of state transitions and channel switching by about $45 \%$ compared with PIP. RSUC increase the network throughput by about $30 \%$ compared with waiting for all the $\mathrm{CH}$ s to complete intercluster communication before entering intracluster communication.

Network delay of emergency data is not considered in RSUC. In future research, IoT resource scheduling based on unequal clustering and priority will be studied.

\section{Data Availability}

The data used to support the findings of this study are available from the corresponding author upon request.

\section{Conflicts of Interest}

The authors declare that they have no conflicts of interest.

\section{Acknowledgments}

This research was partly funded by the National Natural Science Foundation of China (61971031) and the Scientific Research Projects of Ordos Institute of Technology (KYZD2021002).

\section{References}

[1] P. S. S. de Souza, F. P. Rubin, R. Hohemberger et al., "Detecting abnormal sensors via machine learning: an IoT farming WSN-based architecture case study," Measurement, vol. 164, p. 108042, 2020.

[2] Y. Xiao, M. Krunz, and T. Shu, "Multi-operator network Sharing for massive IoT," IEEE Communications Magazine, vol. 57, no. 4, pp. 96-101, 2020.

[3] N. Gupta, M. Khosravy, N. Patel et al., "Economic data analytic AI technique on IoT edge devices for health monitoring of agriculture machines," Applied Intelligence, vol. 50, no. 11, pp. 3990-4016, 2020.

[4] O. Friha, M. A. Ferrag, L. Shu, L. Maglaras, and X. Wang, "Internet of things for the future of smart agriculture: a comprehensive Survey of emerging Technologies," IEEE/CAA Journal of Automatica Sinica, vol. 8, no. 4, pp. 718-752, 2021.

[5] G. Suciu, C. I. Istrate, and M. C. Ditu, "Secure smart agriculture monitoring technique through isolation," in Proceedings of the 2019 Global IoT Summit (GIoTS), June 2019.

[6] T. Kim, D. Qiao, and W. Choi, "Energy-efficient scheduling of Internet of Things devices for environment monitoring applications," in Proceedings of the 2018 IEEE International Conference on Communications (ICC), May 2018.

[7] O. Yang and Y. Wang, "Optimization of time and power resources allocation in communication systems under the industrial internet of things," IEEE Access, vol. 8, pp. 140392-140398, 2020. 
[8] X. Liu, Y. Gao, and F. Hu, "Optimal time scheduling scheme for wireless powered ambient backscatter communications in IoT networks," IEEE Internet of Things Journal, vol. 6, no. 2, pp. 2264-2272, 2019.

[9] Z. Cui, X. Jing, P. Zhao, W. Zhang, and J. Chen, "A new Subspace clustering strategy for AI-based data analysis in IoT system," IEEE Internet of Things Journal, vol. 8, no. 16, pp. 12540-12549, 2021.

[10] A. Aher, J. Kasar, P. Ahuja et al., "Smart agriculture using clustering and IOT," International Research Journal of Engineering and Technology (IRJET), vol. 5, no. 03, pp. 23950056, 2018.

[11] Y. Xue, X. Chang, S. Zhong, and Y. Zhuang, "An efficient energy hole alleviating algorithm for wireless sensor networks," IEEE Transactions on Consumer Electronics, vol. 60, no. 3, pp. 347-355, 2014.

[12] Z. Wang, D. Liu, and A. Jolfaei, "Resource allocation solution for sensor networks using improved chaotic firefly algorithm in IoT environment," Computer Communications, vol. 156, pp. 91-100, 2020.

[13] G. Lee and J. Youn, "Group-based transmission scheduling scheme for Building LoRa-based massive IoT," in Proceedings of the 2020 International Conference on Artificial Intelligence in Information and Communication (ICAIIC), pp. 583-586, IEEE, Fukuoka, Japan, Feb. 2020.

[14] J.-H. Lee and S. H. Cho, "Tree TDMA MAC algorithm using time and frequency slot allocations in tree-based WSNs," Wireless Personal Communications, vol. 95, no. 3, pp. 25752597, 2017.

[15] W. Osamy, A. A. El-Sawy, and A. M. Khedr, "Effective TDMA scheduling for tree-based data collection using genetic algorithm in wireless sensor networks," Peer-to-Peer Networking and Applications, vol. 13, no. 3, pp. 796-815, 2019.

[16] J. S. Kumar and M. A. Zaveri, "Clustering for collaborative processing in IoT network," in Proceedings of the Second International Conference on IoT in Urban Space, pp. 95-97, ACM, New Yark, USA, May 2016.

[17] J. S. Kumar and M. A. Zaveri, "Hierarchical clustering for dynamic and heterogeneous Internet of Things," Procedia Computer Science, vol. 93, pp. 276-282, 2016.

[18] M. A. Eshaftri, A. Y. Aldubai, I. Romdhani, and M. B. Yassien, "An efficient dynamic load-balancing aware protocol for wireless sensor networks," ACM, in Proceedings of the 13th International Conference on Advances in Mobile Computing and Multimedia, pp. 189-194, Singapore, December 2015.

[19] H. Bai, X. Zhang, and F. Ma, "Unequal clustering and routing algorithm based on dynamic topology for WSN," in Proceedings of the 2018 IEEE 4th International Conference on Computer and Communications(ICCC), pp. 311-316, IEEE, Chengdu, China, Dec. 2018.

[20] S. Kumar and Z. Raza, "Using clustering approaches for response time aware job scheduling model for internet of things (IoT)," International Journal of information technology, vol. 9, no. 2, pp. 177-195, 2017.

[21] F. Alam, R. Mehmood, I. Katib, N. N. Albogami, and A. Albeshri, "Data fusion and IoT for smart Ubiquitous environments: a Survey," IEEE Access, vol. 5, pp. 9533-9554, 2017.

[22] T.-T. Huynh, A.-V. Dinh-Duc, and C.-H. Tran, "Delayconstrained energy-efficient cluster-based multi-hop routing in wireless sensor networks," Journal of Communications and Networks, vol. 18, no. 4, pp. 580-588, 2016.

[23] C. Sivaraj, P. J. A. Alphonse, and T. N. Janakiraman, "Independent neighbour set based clustering algorithm for routing in wireless sensor networks," Wireless Personal Communications, vol. 96, no. 4, pp. 6197-6219, 2017.

[24] S. Sankar, S. Ramasubbareddy, A. K. Luhach, A. Nayyar, and B. Qureshi, "CT-RPL: cluster tree based routing protocol to maximize the lifetime of Internet of Things," Sensors, vol. 20, no. 20, p. 5858, 2020.

[25] S. Sankar, S. Ramasubbareddy, F. Chen, and A. H. Gandomi, "Energy-efficient cluster-based routing protocol in internet of things using swarm intelligence," in Proceedings of the 2020 IEEE Symposium Series on Computational Intelligence (SSCI), pp. 219-224, IEEE, Canberra, ACT, Australia, Dec. 2020.

[26] Y. Xu, Z. Yue, and L. Lv, "Clustering routing algorithm and simulation of internet of things perception layer based on energy balance," IEEE Access, vol. 7, pp. 145667-145676, 2019.

[27] P. Chithaluru, S. Kumar, A. Singh, A. Benslimane, and S. K. Jangir, "An energy-efficient routing scheduling based on fuzzy Ranking scheme for internet of things (IoT)," IEEE Internet of Things Journal, vol. 1, p. 1, 2021.

[28] P. Gazori, D. Rahbari, and M. Nickray, "Saving time and cost on the scheduling of fog-based IoT applications using deep reinforcement learning approach," Future Generation Computer Systems, vol. 110, no. 10, pp. 1098-1115, 2019.

[29] R. E. N. Moraes, W. W. F. dos Reis, H. R. O. Rocha, and D. J. C. Coura, "Power-efficient and interference-free link scheduling algorithms for connected wireless sensor networks," Wireless Networks, vol. 26, no. 5, pp. 3099-3118, 2019.

[30] V. Gabale, K. Chebrolu, B. Raman, and S. Bijwe, "Pip," ACM Transactions on Sensor Networks, vol. 8, no. 4, pp. 1-34, 2012.

[31] Y. Yanhong, Z. Xiaotong, L. Qiong, and L. Wenchao, "Dynamic time division multiple access algorithm for industrial wireless hierarchical sensor networks," China Communications, vol. 10, no. 5, pp. 137-145, 2013.

[32] V. Seedha Devi, T. Ravi, and S. B. Priya, "Cluster based data aggregation scheme for latency and packet loss reduction in WSN," Computer Communications, vol. 149, pp. 36-43, 2020.

[33] S. Abdullah, M. N. Asghar, M. Ashraf, and N. Abbas, "An energy-efficient message scheduling algorithm with joint routing mechanism at network layer in internet of things environment," Wireless Personal Communications, vol. 111, no. 3, pp. 1821-1835, 2020.

[34] H. Bai, X. Zhang, Y. Liu, and Y. Xie, "Resource scheduling based on routing tree and detection matrix for Internet of things," International Journal of Distributed Sensor Networks, vol. 17, no. 3, p. $155014772110038,2021$.

[35] X. Zhang, Q. Luo, L. Cheng, Y. Wan, H. Song, and Y. Yang, "CRTRA: coloring route-tree based resource allocation algorithm for industrial wireless sensor networks," in Proceedings of the 2012 IEEE Wireless Communications and Networking Conference (WCNC), pp. 1870-1875, IEEE, Paris, France, April 2012. 